\title{
Application and Development of Zigbee Technology for Smart Grid Environment
}

\author{
Sheng-Fa Chang1, Chi-Feng Chen'1, Jyh-Horng Wen'1, Jih-Hsin Liu'1, Jun-Hong Weng1, \\ Jian-Li Dong2 \\ ${ }^{1}$ Department of Electrical Engineering, Tunghai University, Taichung, Taiwan \\ ${ }^{2}$ Bureau of Standards, Metrology and Inspection (BSMI), Ministry of Economic Affairs, Taiwan \\ Email: g02360024@thu.edu.tw
}

Received December 2014

\begin{abstract}
In the next few years, the home energy management system trend of the smart home will become increasingly evident. From the application and development of recent years, the Bluetooth and Zigbee are the most valued by the industry. Of these two kinds of technology, Zigbee supports low cost and more number of nodes, and with its low power consumption, it is bound to become a major communication feature for the smart home technology. Because Zigbee SEP2.0 specification support multiple network technology features, a product can become compatible with a variety of communication methods to connect with the home network and achieve home automation of functional requirements, facilitating maximum performance of wireless applications.
\end{abstract}

\section{Keywords}

Electromagnetic Compatibility (EMC), Homeplug, Smart Grid, Zigbee

\section{Introduction}

In modern society, the Internet is an essential element in life like water and electricity. With technological development, people no longer just use interconnection equipment for computers. Household appliances, medical instruments, watches and other devices also need interconnection, which is achieved through short-range wireless transmission technology. Other common technologies are infrared, Bluetooth, ZigBee, radio frequency identification (RFID), and near Field communication (NFC).

With the advent of the age of information technology and consumer demand for high quality and reliability, power consumption has also increased. At the same time, the pressure to utilize natural resources and the environment is also increasing rapidly. Smart grid is a system that combines physical power systems and information system, which is connected to a variety of devices and information to form an information platform. Smart grid may add some new technologies in communications, distributed systems, advanced metering, automated storage, etc., allow a substantial increase in smart home network reliability and robustness, and reduce energy consumption and unnecessary waste of resources.

With the advent of the smart grid, consumers can reduce their home electricity costs and energy consumption. For this purpose, some related technologies have been proposed [1]-[8]. To use energy management system in

How to cite this paper: Chang, S.-F., Chen, C.-F., Wen, J.-H., Liu, J.-H., Weng, J.-H. and Dong, J.-L. (2015) Application and Development of Zigbee Technology for Smart Grid Environment. Journal of Power and Energy Engineering, 3, 356-361. 
residential areas, smart home devices are used widely. However, for existing appliances, we need to add a data processor and a wireless data transceiver device in every home. The data processor may analyze the received data transceiver and allow appropriate operation of the device. These types of home devices have emerged. Some smart air conditioners can be interconnected with a tablet PC or a mobile phone and can be controlled by the user. If every household device can be interconnected, each user will be able to centrally control the devices and reduce energy consumption, thus achieving the goal of a smart home.

\section{Smart Grid and Zigbee SEP2.0}

\subsection{Smart Grid Drivers}

The key market drivers behind Smart Grid solutions are [9]:

- Need for more energy.

- Increased usage of renewable energy resources.

- Sustainability.

- Competitive energy prices.

- Security of supply.

- Ageing infrastructure and workforce.

The utilities have to master the following challenges:

- High power system loading.

- Increasing distance between generation and load.

- Fluctuating renewable.

- New loads (hybrid/e-cars).

- Increased use of distributed energy resources.

- Cost pressure.

- Utility unbundling.

- Increased energy trading.

- Transparent consumption and pricing for the consumer.

- Significant regulatory push.

Smart Energy Profile 2.0 [10] can satisfy the functional requirements of the Neighborhood Area Network and Home Area Network, and provide home automation control, advanced meter reading system (Advanced Metering infrastructure, AMI) and wisdom metering system (Smart Metering) applications, as shown in Figure 1.

With the development of standardization in SEP2.0, it has been proven to be compatible with multiple network technologies. There is rapid development of this SEP2.0 in the United States. To promote smart grid standardization bodies (National Institute of Standards and Technology, NIST) and to develop wireless communication

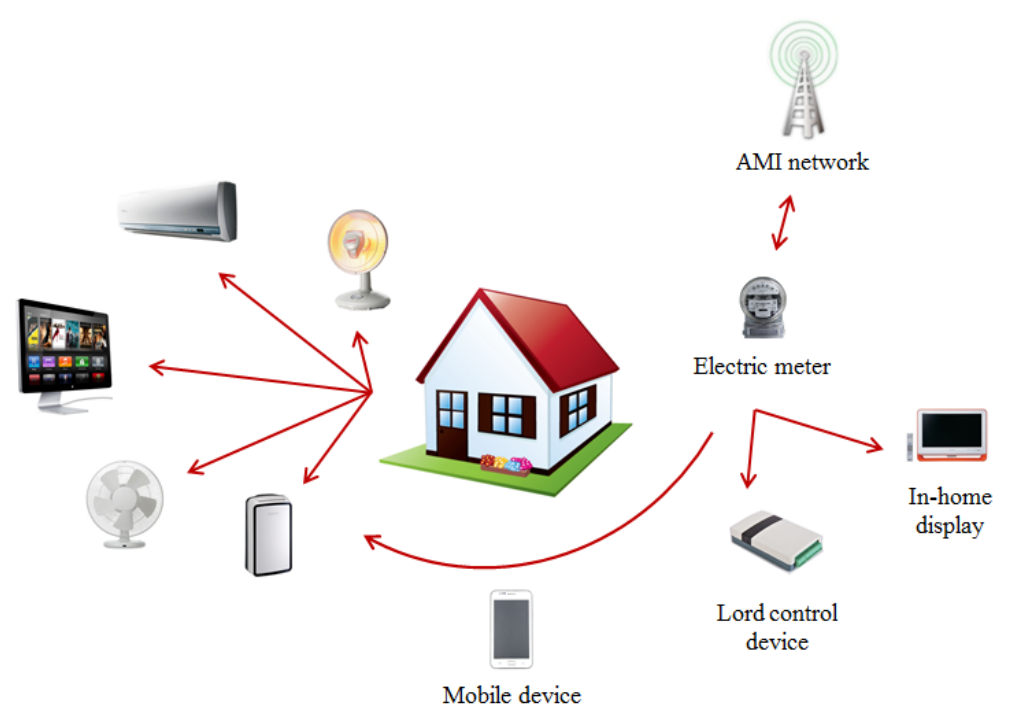

Figure 1. Smart grid architecture. 
standards for residential appliances, the standard US smart grid interoperability standards framework was completed in 2009 (Initial Smart Grid Interoperability Standards Framework) and version 1.0 is used in next-generation grid plans.

\subsection{Zigbee Smart Energy Profile 2.0 Features}

Smart Energy Profile 2.0 (SEP2.0) adopts the IPv6 architecture and the use of communications technology entities will no longer be limited to the IEEE 802.15.4. It supports IEEE 802.11. Power-line technology, as shown in Figure 2, is a breakthrough in the development of network management and features original number of devices and the transmission speed of the problem, effective power systems and energy use in series end, and the smart to meet the power system control function planning.

SEP2.0 supports the same physical layer (Physical Layer, PHY) specifications for a variety of network, such as wireless LAN (Wi-Fi) and Power Line Communication (PLC), etc., and can communicate with each standard room, and further expand the smart grid control, as shown in the following functional description of each layer. SEP2.0 layers are shown in Figure 3.

\section{Link Layer:}

SEP2.0 is designed to operate in any Link layer technology, and has the following features:

1) Support for all possible physical layers, e.g., IEEE 802.15.4, 802.11 series, 802.3 series, HomePlug and IEEE P1901.

2) Support wireless communication and power lines wired Homeplug in ESI.

3) Support wired home network (Home Area Network, Han) and bridging of the wireless home network, and maintain two different kinds of network compatibility.

4) In the Energy Services Interface (Energy Service Interface, ESI), support HomePlug IEEE802.15.4 and physical layer interface.

\section{Adaptation Layer:}

SEP2.0 is designed to run on the IP network. Therefore, it should be able to run on any underlying network, except for some specific network environment, in order to achieve greater efficiency, performance or reliability. For interoperability reasons, Adaptation on the layer structure must have consistency.

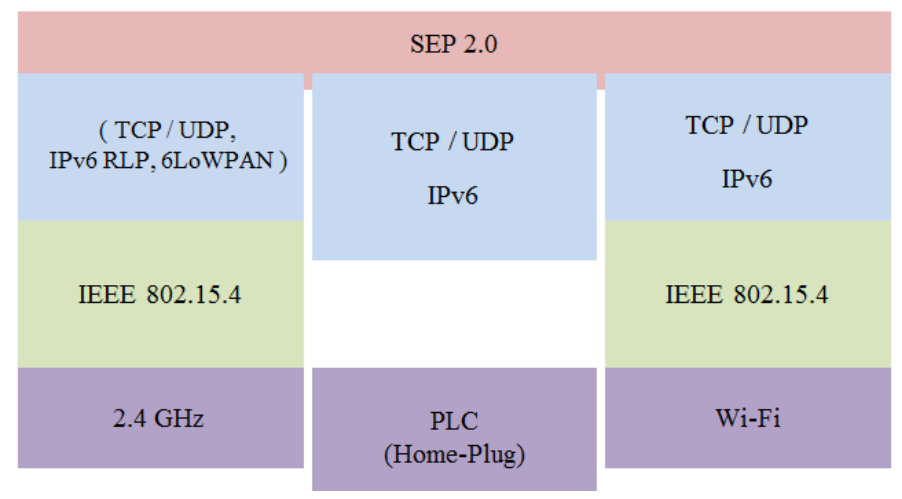

Figure 2. Zigbee SEP2.0 communication layer architecture.

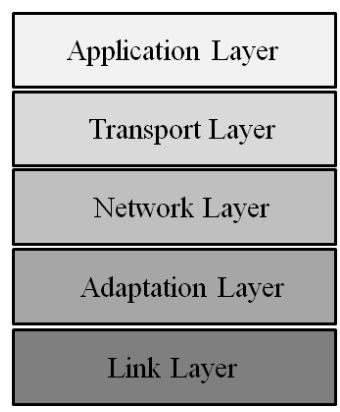

Figure 3. Zigbee SEP 2.0 communication model. 
1) To run on 802.15.4, the 6LoWPAN architecture (IPv6 over Low Power Wireless Personal Area Networks) must be used.

2) To run on the power line network, IEEE 802.2 and IPv6 architecture should be running.

3) In addition to two or more networks, to run on the remaining network, it must comply with the MAC IETF Recommendation RFC4919 standards as defined in the PHY layer.

Network Layer:

SEP2.0 must be used for IPv6 on the Network layer network communications, contrary to all the various energy-saving equipment. SEP2.0 must support the IPv6 architecture (architecture includes the address, address auto-configuration, Neighbor Discovery function).

Transport Layer:

Because SEP2.0 supports HTTP, it also supports the use of the Transport layer UDP (User Datagram Protocol) and TCP (Transmission Control Protocol).

\section{Application Layer:}

SEP2.0 in the Application layer is divided into two parts, namely business objective technical requirements and supporting technical requirements. Business objective technical requirements for functionality of SEP2.0 require basic settings; it is recommended that the service equipment have this function. The supporting technical requirements are for the business objective technical requirements, which are subdivided to provide intelligent energy standard operating environment necessary for management capabilities. The following section explains this two-part features and the meaning of their functions.

\section{Zigbee SEP 2.0 Applications}

Zigbee was applied in the early development of automation and control, such as environmental monitoring, industrial automation, etc. Global climate anomalies, decreasing energy, aging population and other issues have prompted the development of networking in home automation (smart home), and wisdom meter and other applications have become increasingly important.

ZigBee Alliance realizes the wisdom of energy management, and it hopes to develop the specification standards for manufacturers of related products such as meter (Smart Meter), home energy monitor (In-home Display), load controller (Load Controller), and energy gateways (Gateway) so they can communicate; compared with SEP 1.0, SEP 2.0 supports a broader level, such as the use of IPv6 architecture, supports IEEE 802.11 (Wi-Fi), Powerline (PLC) and other communication technologies to facilitate energy consumption in devices in series with the power system.

From the application perspective, short-range wireless transmission technology development is a trend not just for consumers but also for medical care, wearable devices, mobile payments, and finally almost every smart mobile devices. In order to add more value, these front-end devices for consumer use may require more than one wireless technology; in addition, size, power consumption and manufacturing costs and other factors are considered. Thus, integrating a variety of wireless communication technologies in a single chip has gradually become popular.

\section{EMC in Smart Grid}

Electromagnetic compatibility (EMC) in the smart grid is very important. With reference to the basic EMC standards, it has detailed measurement and test methods, EMC generic standard specifies the limited number of essential emission and immunity testing, and the lowest level of the test. The aim is to ensure a good balance of technical and economic considerations of the compatibility.

The key to smart grid is that it has a "two-way communication" function; the power companies use remote monitoring systems, understand the electrical conditions around the country, and the deployment of power. The users can also keep track of their electricity situation, further regulate power consumption, and achieve energy-saving and money-saving effects.

The basic structure of smart grid is the advanced metering infrastructure (Advanced Metering Infrastructure, AMI), which is a very important part. AMI is the smart of the meter, two-way communication networks and information analysis management and controls software, electrical safety protection mechanism and automatic meter reading control center.

The domestic industry is also actively expanding its products using the smart grid architecture, and while there is a need to rely on foreign countries to perform the test, there have been smart grid related equipment 
Table 1. Test items.

\begin{tabular}{ll}
\hline \multicolumn{1}{c}{ EMI test } & EMS test \\
\hline$\bullet$ Conducted Emission & $\bullet$ ESD \\
$\bullet$ & $\bullet$ RS \\
& $\bullet$ Surge \\
& $\bullet$ CS \\
& $\bullet$ LFCS \\
& $\bullet$ PFMF \\
& $\bullet$ OSC-MF \\
& $\bullet$ OSC-wave \\
& $\bullet$ AC-DIP \\
& $\bullet$ DC-DIP \\
& $\bullet$ Ripper \\
& $\bullet$ SWC \\
\hline
\end{tabular}

manufacturers, which provide exceptional EMC testing in the energy industry in the country, test items are shown in Table 1.

Services include smart grid applied to all kinds of electronic products, such as the smart of the meter, telecommunications equipment, information technology equipment used within the plant, and wireless (RF) devices.

\section{Conclusion}

In recent years, smart grid has become popular, and the application of the future will be short-range wireless transmission of smart family wearable electronic device. If combined with Zigbee SEP2.0 standardized features to support multiple network technologies, and using EMC electromagnetic interference and electromagnetic tolerance test, the surface of the product will have characteristics compatible with a variety of communications to achieve the home networking and home automation functional requirements.

\section{Acknowledgements}

The authors would like to thank research group from BSMI (Bureau of Standards, Metrology and Inspection) for their technical support. This work was supported by the BSMI under Contract No. 1D151031201-117, grant from BSMI, Taiwan.

\section{References}

[1] Zhao, Z., Lee, W.C., Shin, Y. and Song, K. (2013) An Optimal Power Scheduling Method for Demand Response in Home Energy Management System. IEEE Transactions on Smart Grid, 4, 1391-1400. http://dx.doi.org/10.1109/TSG.2013.2251018

[2] White, C.B. (2012) Critical EMC Test Issues Needing Early Resolution. IEEE International Symposium on Electromagnetic Compatibility, Pittsburgh, 6-10 August 2012, 127-131. http://dx.doi.org/10.1109/ISEMC.2012.6351797

[3] Cataliotti, A., Cara, D., Marsala, G., Ragusa, A. and Tinè, G. (2013) Electromagnetic Immunity Analysis of a New Interface Device with Power Line Communication for Smart Grid and Energy Storage Applications. IEEE 17th International Symposium on Power Line Communications and Its Applications, Johannesburg, 24-27 March 2013, 214-219.

[4] Keebler, P. (2012) Meshing Power Quality and Electromagnetic Compatibility for Tomorrow's Smart Grid. IEEE Electromagnetic Compatibility Magazine, 1, 100-103. http://dx.doi.org/10.1109/MEMC.2012.6244982

[5] Olofsson, M. (2009) Power Quality and EMC in Smart Grid. EPQU 10th International Conference on Electrical Power Quality and Utilisation, Lodz, 15-17 September 2009, 1-6. http://dx.doi.org/10.1109/EPQU.2009.5318811

[6] Liu, Y., Ren, W., Feng, J. and Hu, X. (2012) Research on Electromagnetic Compatibility Test Port Model of Smart High Voltage Equipment. 6th Asia-Pacific Conference on Environmental Electromagnetics, Shanghai, 6-9 November 2012, 381-383.

[7] Maas, J. (2012) Smart Grid and Electrostatic Discharge: Cause for New Concerns? International Symposium on Electromagnetic Compatibility (EMC EUROPE), Italy, 17-21 September 2012, 1-6. 
http://dx.doi.org/10.1109/EMCEurope.2012.6396772

[8] Heirman, D. (2012) What Makes Smart Grid-Smart—And Who Is in the Game? IEEE Electromagnetic Compatibility Magazine, 1, 95-99.

[9] SMB Smart Grid Strategic Group (SG3) (2010) IEC Smart Grid Standardization Roadmap.

[10] ZigBee-095449 DRAFT (2009) ZigBee Smart Energy Profile 2.0 Technical Requirements Document. 